\title{
SINTOMAS DE DEFICIÊNCIA NUTRICIONAL EM MUDAS DE Acacia holosericea EMRESPOSTA À OMISSÃO DE MACRONUTRIENTES ${ }^{1}$
}

\author{
Tathiane Santi Sarcinelli², Emerson Silva Ribeiro Jr. ${ }^{2}$, Luiz Eduardo Dias ${ }^{2,3}$ e Leila de Souza Lynch ${ }^{2}$
}

\begin{abstract}
RESUMO - A Acacia holosericea é uma espécie leguminosa arbórea bastante utilizada na recuperação de áreas degradadas. O conhecimento dos sintomas de deficiência nutricional apresentados por esta espécie possibilita a identificação e a correção de deficiências em exemplares plantados em substratos degradados. Os objetivos deste trabalho foram caracterizar a sintomatologia visual de carências de macronutrientes e avaliar a produção de biomassa e o acúmulo de nutrientes nas raízes e na parte aérea de mudas de Acacia holosericea, submetidas a diferentes soluções nutritivas com exclusão de macronutrientes. Os tratamentos constituíram-se de sete soluções nutritivas: 1) solução completa (SC); 2) SC -N; 3) SC -P; 4) SC -K; 5) SC -Ca; 6) SC -Mg; e 7) SC -S. Os tratamentos -N e -Mg foram os que mais afetaram a produção total de biomassa. $\mathrm{O}$ decréscimo de produção manifestou-se na seguinte ordem: $-\mathrm{N}=-\mathrm{Mg}>-\mathrm{K}>-\mathrm{S}>-\mathrm{Ca}>\mathrm{SC}>-\mathrm{P}$. As plantas do tratamento $-\mathrm{N}$ formaram nódulos no sistema radicular, exibindo teores foliares de $\mathrm{N}$ maiores que as plantas do tratamento SC. Com exceção dos tratamentos SC e -P, todos os outros apresentaram sintomas de deficiência. A ausência de $\mathrm{S}$ alterou a disposição natural dos filódios novos da A. holosericea.
\end{abstract}

Palavras-chave: Leguminosas arbóreas, recuperação de áreas degradadas, nutrição de plantas e Acacia holosericea.

\section{SYMPTOMS OF NUTRITIONAL DEFICIENCY IN SEEDLINGS OF Acacia holosericea SUBMITTED TO ABSENCE OF MACRONUTRIENTS}

\begin{abstract}
Acacia holosericea is a leguminous species that has been extensively used in land reclamation programs. The knowledge of its nutritional symptoms allows the identification and correction of deficiencies in individuals planted in degraded substrates. The aim of this work was to characterize the behavior of seedlings of A. holosericea submitted to different nutritional solutions with absence of macronutrients. The treatments consisted of seven nutritional solutions: 1) Complete solution (SC); 2) SC - N; 3) SC-P;4) SC-K; 5) SC-Ca; 6) SC-Mg; 7) $S C-S$. Treatments $-N$ and $-M g$ were the ones that most affected biomass production. Decrease in biomass production occurred in the following order: $-N=-M g>-K>-S>-C a>S C>-P$. The $-N$ treatment plants formed nodules in their roots, showing higher concentrations of $N$ than those of the SC treatment. Except for the SC and-P treatments, all the other treatments showed nutritional deficiency symptoms. The absence of $S$ has led to a change in the natural disposition of new leaves of A. holosericea.
\end{abstract}

Key words: $\quad$ Leguminous trees, land reclamation, plant nutrition and Acacia holosericea.

\section{INTRODUÇÃO}

O uso de leguminosas arbóreas em programas de recuperação de áreas degradadas $(\mathrm{RAD})$ por mineração tem sido adotado com bons resultados em diferentes condições ambientais (Franco et al., 1989, 1992). Esta técnica é baseada na elevada capacidade de adaptação e produção de biomassa de diferentes espécies desta família. Devido à capacidade de associação com bactérias fixadoras de nitrogênio atmosférico e com fungos

Recebido para publicação em 16.3.2003 e aceito para publicação em 30.4.2004.

Departamento de Solos, Universidade Federal de Viçosa, 36571-000 Viçosa-MG, Tel.: (31) 3899-2630; ${ }^{3}$ Pesquisador CNPq. 
micorrízicos, significativa parcela dessas espécies pode potencializar estas qualidades, tornando-as importantes para programas de RAD.

A Acacia holosericea A. Cunn. ex G. Don é uma espécie leguminosa arbórea de ocorrência natural na Austrália e que vem sendo cultivada no Brasil já há alguns anos. Na fase inicial de crescimento apresenta folhas compostas, característica comum da família Leguminosae. À medida que a planta cresce, seu pecíolo dilata e as folhas compostas caem, deixando uma cicatriz na extremidade do pecíolo dilatado, denominado filódio, que passa a exercer a função de folha. Além dessa interessante característica morfológica, esta espécie apresenta qualidades importantes para programas de recuperação ambiental. Elevadas concentrações de matéria orgânica em substratos cultivados com Acacia holosericea refletem sua grande capacidade de produção de biomassa, queda de filódios e, principalmente, produção de raízes, características desejáveis para espécies a serem utilizadas na revegetação de áreas degradadas (Franco et al., 1992; Dias et al., 1999).

O Departamento de Solos da Universidade Federal de Viçosa vem desenvolvendo um programa de pesquisa com espécies potencialmente interessantes para RAD. Diferentes estudos têm sido conduzidos com o objetivo de avaliar a tolerância de espécies leguminosas arbóreas e arbustivas a substratos compactados (Campello, 1998), ácidos e salinos, como também as exigências em P, K e S (Dias et al., 1990, 1991a, 1991b, 1992) e os sintomas de deficiência em macronutrientes (Balieiro, 2001).

O conhecimento de sintomas de deficiência nutricional em espécies utilizadas em RAD permite a identificação e correção de deficiências que podem ocorrer em exemplares plantados em diferentes substratos degradados, possibilitando, desta maneira, intervenções corretas, sem desperdícios e de menor impacto ambiental.

Os objetivos deste trabalho foram caracterizar a sintomatologia visual de carências de macronutrientes e avaliar a produção de biomassa e o acúmulo de nutrientes nas raízes e na parte aérea de mudas de Acacia holosericea, submetidas a diferentes soluções nutritivas com exclusão de macronutrientes.

\section{MATERIAL EMÉTODOS}

Sementes de Acacia holosericea, provenientes da região de Porto Trombetas - PA, foram escarificadas com ácido sulfúrico concentrado por 8 minutos, lavadas e postas para germinar em leito de areia lavada e tratada com $\mathrm{HCl}$ 0,1 Mol/1. As plantas foram mantidas em leito de areia durante três meses, quando foram transferidas para vasos de 1 litro, contendo o mesmo substrato. Durante dois meses as plantas continuaram a receber a solução nutritiva com todos os macronutrientes, segundo Dias et al. (1994), e solução de micronutrientes, segundo Ruiz (1986). Findo este período, as plantas passaram a receber aplicações semanais das diferentes soluções nutritivas referentes aos sete tratamentos do experimento: 1) solução completa (SC); 2) solução com omissão de nitrogênio (SC -N); 3) solução com omissão de fósforo (SC -P); 4) solução com omissão de potássio (SC -K); 5) solução com omissão de cálcio (SC -Ca); 6) solução com omissão de magnésio (SC -Mg); 7) solução com omissão de enxôfre (SC -S) (Quadro 1). Estas soluções foram aplicadas durante seis meses.

As plantas foram monitoradas quanto ao aparecimento de sintomas de deficiências nutricionais. Quando do aparecimento, estes foram descritos e fotografados, tendo os filódios afetados sido analisados em detalhe.

Após os seis meses da aplicação dos tratamentos, as plantas foram cortadas rente ao substrato e avaliadas quanto à produção de filódios novos e velhos, ramos + caule e raízes. As diferentes partes das plantas foram submetidas à digestão nítrico-perclórica (Blanchar et al., 1963), para dosagem dos teores totais de $\mathrm{Ca}$ e $\mathrm{Mg}$ por espectrometria de absorção atômica, K por fotometria de chama, S por turbimetria (Alvarez V., 2001) e P por colorimetria (Braga \& Defelipo, 1974). Os teores de N foram determinados pelo método semimicro Keljdhal (Jones et al., 1991).

Quadro 1 - Concentração de nutrientes nas soluções nutritivas utilizadas em cada tratamento 1 /

Table 1 - Nutrient concentration in the nutritive solutions applied in each treatment $\underline{1}$

\begin{tabular}{|c|c|c|c|c|c|c|}
\hline \multirow{2}{*}{ Tratamento } & $\mathrm{N}$ & $\mathrm{P}$ & $\mathrm{K}$ & $\mathrm{Ca}$ & $\mathrm{Mg}$ & $\mathrm{S}$ \\
\cline { 2 - 7 } & \multicolumn{7}{|c|}{$(\mathrm{mmol} / \mathrm{l})$} \\
\hline $\mathrm{SC}^{2} !$ & 5,5 & 0,7 & 2,0 & 1,4 & 0,3 & 0,3 \\
\hline$-\mathrm{N}$ & - & 0,7 & 2,0 & 1,4 & 0,3 & 0,3 \\
\hline$-\mathrm{P}$ & 5,5 & - & 2,0 & 1,4 & 0,3 & 0,3 \\
\hline$-\mathrm{K}$ & 5,5 & 0,7 & - & 1,4 & 0,3 & 0,3 \\
\hline$-\mathrm{Ca}$ & 5,5 & 0,7 & 2,0 & - & 0,3 & 0,3 \\
\hline$-\mathrm{Mg}$ & 5,5 & 0,7 & 2,0 & 1,4 & - & 0,3 \\
\hline$-\mathrm{S}$ & 5,5 & 0,7 & 2,0 & 1,4 & 0,3 & - \\
\hline
\end{tabular}

${ }^{1 /}$ Micronutrientes aplicados a todos os tratamentos (Ruiz, 1986).

²/ Solução nutritiva completa. 
Os valores de matéria seca de parte aérea e de raízes e os teores e conteúdos de macronutrientes nas diferentes partes das plantas foram submetidos à análise de variância e os valores médios comparados por meio do teste de Tukey, a 5\% de probabilidade.

O experimento foi conduzido em casa de vegetação da Universidade Federal de Viçosa - UFV, em um delineamento inteiramente casualizado, com quatro repetições, perfazendo um total de 28 unidades experimentais, constituídas por vasos com uma planta cada.

\section{RESULTADOS E DISCUSSÃO}

\subsection{Produção de Biomassa, Teores e Conteúdos de Nutrientes nos Tecidos}

A omissão de $\mathrm{P}$ e de Ca proporcionou a formação de uma biomassa vegetal total que não diferiu estatisticamente daquela apresentada pelas plantas submetidas ao tratamento com todos os nutrientes (Quadro 2). O decréscimo de produção ocorreu na seguinte ordem: -N $=-\mathrm{Mg}>-\mathrm{K}>-\mathrm{S}>-\mathrm{Ca}>\mathrm{SC}>-\mathrm{P}$. Em um estudo realizado com outra espécie do mesmo gênero (A. mangium), Dias et al. (1990) constataram baixas exigências de Ca para a formação de mudas da espécie.

As maiores reduções na produção de biomassa total foram verificadas para as plantas submetidas aos tratamentos - $\mathrm{N} \mathrm{e} \mathrm{-Mg} \mathrm{(Quadro} \mathrm{2).} \mathrm{As} \mathrm{plantas} \mathrm{que} \mathrm{receberam}$

Quadro 2 - Produção de matéria seca dos filódios (MSF), dos ramos + caule (MSRC), da parte aérea (MSPA), das raízes (MSR) e total (MST) de Acacia holosericea, em cada tratamento

Table 2 - Dry matter production of leaves (MSF), branches + stem $(M S R C)$, shoots (MSPA), roots (MSR) and total (MST) of Acacia holosericea plants, in each treatment

\begin{tabular}{|c|c|c|c|c|c|}
\hline \multirow{2}{*}{$\begin{array}{l}\text { Trata- } \\
\text { mento }\end{array}$} & MSF & MSRC & MSPA & MSR & MST \\
\hline & \multicolumn{5}{|c|}{ (g/vaso) } \\
\hline $\mathrm{SC}$ & $23,4 \mathrm{AB}$ & $15,5 \mathrm{AB}$ & $38,9 \mathrm{AB}$ & $14,1 \mathrm{~A}$ & $53,1 \mathrm{AB}$ \\
\hline$-\mathrm{N}$ & $15,0 \mathrm{BC}$ & $8,7 \mathrm{CD}$ & $23,7 \mathrm{C}$ & $5,5 \mathrm{BC}$ & $29,2 \mathrm{D}$ \\
\hline$-\mathrm{P}$ & $28,3 \mathrm{~A}$ & $21,4 \mathrm{~A}$ & $49,7 \mathrm{~A}$ & $13,3 \mathrm{~A}$ & $63,1 \mathrm{~A}$ \\
\hline$-\mathrm{K}$ & $17,9 \mathrm{BC}$ & $10,6 \mathrm{BCD}$ & $28,5 \mathrm{BC}$ & $8,5 \mathrm{ABC}$ & $36,9 \mathrm{CD}$ \\
\hline$-\mathrm{Ca}$ & $22,7 \mathrm{AB}$ & $16,1 \mathrm{AB}$ & $38,8 \mathrm{AB}$ & $10,7 \mathrm{AB}$ & $49,6 \mathrm{ABC}$ \\
\hline$-\mathrm{Mg}$ & $19,8 \mathrm{ABC}$ & $5,7 \mathrm{D}$ & $25,6 \mathrm{C}$ & $4,5 \mathrm{C}$ & $30,1 \mathrm{D}$ \\
\hline$-S$ & $13,3 \mathrm{C}$ & $13,8 \mathrm{BC}$ & $27,0 \mathrm{BC}$ & $10,3 \mathrm{AB}$ & $37,3 \mathrm{BCD}$ \\
\hline
\end{tabular}

Valores seguidos da mesma letra nas colunas não diferem significativamente pelo teste $\mathrm{T}$ a $5 \%$ de probabilidade. solução nutritiva sem Mg mostraram uma produção de matéria seca de caule + ramos (MSCR) muito baixa, com um valor de aproximadamente a terça parte do tratamento $\mathrm{SC}$. Os tratamentos $-\mathrm{N}$ e $-\mathrm{Mg}$ obtiveram as menores produções de matéria seca de parte aérea (MSPA), o que evidencia a grande demanda de $\mathrm{N}$ pelas espécies da família Leguminosae (Thompson et al., 1986). A menor produção de matéria seca de raízes (MSR) foi obtida com a aplicação do tratamento $-\mathrm{Mg}$, seguido dos tratamentos -N e -K, respectivamente. Houve nodulação em todas as plantas do tratamento -N, o que corrobora com Siqueira \& Franco (1988), que relataram que o $\mathrm{N}$, dentre todos os nutrientes, é o que tem maior efeito sobre a fixação biológica de $\mathrm{N}(\mathrm{FBN})$, porém esta somente ocorrerá em situações de deficiência deste nutriente. Possivelmente este comportamento possa ser explicado pelo fato de $\mathrm{N} \mathrm{e}$ $\mathrm{Mg}$ tomarem parte da molécula de clorofila, com um átomo de $\mathrm{Mg}$ ocupando a posição central desta, conforme a fórmula empírica da clorofila: $\mathrm{C}_{55} \mathrm{H}_{72} \mathrm{O}_{5} \mathrm{~N}_{4} \mathrm{Mg}$ (Devlin, 1969).

Espécies arbóreas de rápido crescimento tendem a apresentar elevada capacidade de absorção de $\mathrm{P}$ em sua fase inicial de crescimento, podendo, inclusive, apresentar significativo potencial de acúmulo de P-inorgânico no vacúolo das células (Grespan, 1997). O longo período inicial de fornecimento de solução completa (SC) às plantas, possivelmente, permitiu o acúmulo do nutriente e a formação de um estoque que passou a ser utilizado eficientemente pela planta quando houve a supressão do nutriente.

Ao final da condução do experimento, os teores de $\mathrm{P}$ tanto nos filódios novos como nos velhos, observados para as plantas submetidas ao tratamento -P, foram significativamente menores que os observados para as plantas que receberam solução nutritiva completa (Quadro 3). Em termos das quantidades absorvidas de P nos filódios velhos, as plantas submetidas à ausência deste nutriente apresentaram um conteúdo mais de quatro vezes menor que as plantas submetidas ao tratamento SC (Quadro 4). Considerando os diferentes compartimentos da planta, essa diferença aumenta para um valor superior a seis vezes.

Analisando o conteúdo de $\mathrm{Ca}$, pode-se observar que não houve redistribuição interna deste nutriente, uma vez que os filódios novos apresentaram conteúdo de $\mathrm{Ca}$ cerca de dez vezes menor que os filódios velhos, evidenciando uma elevada eficiência de utilização do 
nutriente pela espécie (Quadro 5). Observa-se, ainda, que o conteúdo total de Ca nas plantas submetidas à ausência deste elemento (-Ca) não diferiu significativamente do conteúdo observado nas plantas do tratamento SC.

A elevada eficiência de utilização de Ca também foi observada para a Acacia mangium (Dias et al., 1994), espécie que, assim como a Acacia holosericea, é considerada pioneira e possui grande plasticidade no que se refere à sua capacidade de adaptação a diferentes condições de substrato e clima e que tem apresentado elevado potencial para uso em programas de recuperação ambiental. As plantas submetidas ao tratamento -P apresentaram elevada capacidade de absorção de $\mathrm{Ca}$, o que é deduzido do elevado conteúdo do elemento apresentado pelas plantas deste tratamento, tendo o conteúdo de $\mathrm{Ca}$ na planta inteira sido significativamente maior que em todos os outros tratamentos, igualando-se estatisticamente apenas ao tratamento SC.

$\mathrm{O}$ tratamento $-\mathrm{N}$ apresentou elevado teor de $\mathrm{N}$ nos filódios novos e velhos e que foram significativamente superior aos encontrados nas plantas que receberam solução nutritiva completa (Quadro 3). Este resultado pode ser explicado devido à nodulação que ocorreu no sistema radicular de todas as plantas deste tratamento. A fixação biológica de $\mathrm{N}$ resultou em maior teor de $\mathrm{N}$ na planta do que a absorção de $\mathrm{N}$ fornecido pela solução nutritiva. Desta forma, a omissão de $\mathrm{N}$ possibilitou a formação de simbiose com rizóbios que foram eficientes na formação de nódulos e fixação de $\mathrm{N}_{2}$ atmosférico, o que, por sua vez, resultou em uma maior assimilação deste nutriente.

Quadro 3 - Teores de macronutrientes em filódios novos (FN) e filódios velhos (FV) de plantas de Acacia holosericea, em cada tratamento

Table 3 - Macronutrient concentrations in new leaves (FN) and old leaves (FV) of Acacia holosericea plants, in each treatment

\begin{tabular}{|c|c|c|c|c|c|c|c|c|c|c|c|c|}
\hline \multirow{3}{*}{ Filódio } & \multicolumn{2}{|c|}{$\mathrm{N}$} & \multicolumn{2}{|c|}{$\mathrm{P}$} & \multicolumn{2}{|c|}{$\mathrm{K}$} & \multicolumn{2}{|c|}{$\mathrm{Ca}$} & \multicolumn{2}{|c|}{$\mathrm{Mg}$} & \multicolumn{2}{|c|}{$S$} \\
\hline & $\mathrm{SC}$ & $-\mathrm{N}$ & $\mathrm{SC}$ & $-\mathrm{P}$ & $\mathrm{SC}$ & $-K$ & $\mathrm{SC}$ & $-\mathrm{Ca}$ & $\mathrm{SC}$ & $-\mathrm{Mg}$ & $\mathrm{SC}$ & $-\mathrm{S}$ \\
\hline & \multicolumn{12}{|c|}{$(\mathrm{g} / \mathrm{kg})$} \\
\hline FN & $28,3 \mathrm{~B}$ & $42,2 \mathrm{~A}$ & $1,4 \mathrm{~A}$ & $1,0 \mathrm{~B}$ & $19,6 \mathrm{~A}$ & $2,0 \mathrm{~B}$ & $8,2 \mathrm{~A}$ & $6,5 \mathrm{~B}$ & $1,6 \mathrm{~A}$ & $0,4 \mathrm{~B}$ & $1,1 \mathrm{~A}$ & $\mathrm{ALR}^{1 /}$ \\
\hline FV & $20,0 \mathrm{~B}$ & $27,3 \mathrm{~A}$ & $2,2 \mathrm{~A}$ & $0,4 \mathrm{~B}$ & $18,0 \mathrm{~A}$ & $1,7 \mathrm{~B}$ & $9,4 \mathrm{~A}$ & $8,5 \mathrm{~B}$ & $0,8 \mathrm{~A}$ & $0,4 \mathrm{~B}$ & $0,6 \mathrm{~A}$ & $0,1 \mathrm{~B}$ \\
\hline
\end{tabular}

Valores seguidos da mesma letra nas linhas, para cada macronutriente isoladamente, não diferem significativamente pelo teste $\mathrm{T}$, a $5 \%$ de probabilidade.

1/ALR: Abaixo do limite de resolução da metodologia.

Quadro 4 - Conteúdo de $\mathrm{P}$ nos filódios novos (FN), nos filódios velhos $(\mathrm{FV})$, nos ramos + caule ( $\mathrm{RC})$, nas raízes (RZ) e planta inteira de Acacia holosericea, em cada tratamento

Table 4 - P content in new leaves (FN), old leaves $(F V)$, branches + stem $(R C)$, roots $(R Z)$ and the whole plant of Acacia holosericea, in each treatment

\begin{tabular}{|l|c|c|c|c|c|}
\hline \multirow{2}{*}{$\begin{array}{l}\text { Trata- } \\
\text { mento }\end{array}$} & FN & FV & RC & RZ & Total \\
\cline { 2 - 7 } & \multicolumn{5}{|c|}{$(\mathrm{mg} /$ planta $)$} \\
\hline $\mathrm{SC}$ & $3,1 \mathrm{~A}$ & $45,2 \mathrm{~B}$ & $48,0 \mathrm{~A}$ & $49,7 \mathrm{~A}$ & $146,0 \mathrm{~A}$ \\
\hline$-\mathrm{N}$ & $3,1 \mathrm{~A}$ & $64,6 \mathrm{AB}$ & $38,5 \mathrm{AB}$ & $23,2 \mathrm{~B}$ & $129,3 \mathrm{~A}$ \\
\hline$-\mathrm{P}$ & $1,6 \mathrm{~A}$ & $11,2 \mathrm{C}$ & $5,7 \mathrm{D}$ & $5,2 \mathrm{C}$ & $23,6 \mathrm{~B}$ \\
\hline$-\mathrm{K}$ & $2,8 \mathrm{~A}$ & $60,6 \mathrm{AB}$ & $32,8 \mathrm{BC}$ & $22,8 \mathrm{~B}$ & $119,1 \mathrm{~A}$ \\
\hline$-\mathrm{Ca}$ & $4,0 \mathrm{~A}$ & $68,8 \mathrm{AB}$ & $48,8 \mathrm{~A}$ & $30,7 \mathrm{~B}$ & $152,3 \mathrm{~A}$ \\
\hline$-\mathrm{Mg}$ & $2,9 \mathrm{~A}$ & $64,7 \mathrm{AB}$ & $23,5 \mathrm{C}$ & $15,0 \mathrm{BC}$ & $106,1 \mathrm{~A}$ \\
\hline$-\mathrm{S}$ & $2,9 \mathrm{~A}$ & $78,4 \mathrm{~A}$ & $41,6 \mathrm{AB}$ & $29,3 \mathrm{~B}$ & $152,2 \mathrm{~A}$ \\
\hline
\end{tabular}

Valores seguidos da mesma letra nas colunas não diferem significativamente pelo teste $\mathrm{T}$, a $5 \%$ de probabilidade.
Quadro 5 - Conteúdo de Ca nos filódios novos (FN), nos filódios velhos (FV), nos ramos + caule ( $\mathrm{RC})$, nas raízes (RZ) e planta inteira de Acacia holosericea, em cada tratamento

Table 5 - Ca content in new leaves (FN), old leaves $(F V)$ branches + stem $(R C)$, roots $(R Z)$ and the whole plant of Acacia holosericea, in each treatment

\begin{tabular}{|c|c|c|c|c|c|}
\hline \multirow{2}{*}{$\begin{array}{c}\text { Trata- } \\
\text { mento }\end{array}$} & FN & FV & RC & RZ & Total \\
\cline { 2 - 6 } & \multicolumn{5}{|c|}{$(\mathrm{mg} /$ planta) } \\
\hline SC & $18,9 \mathrm{~A}$ & $198,4 \mathrm{~B}$ & $179,3 \mathrm{AB}$ & $139,8 \mathrm{~A}$ & $536,3 \mathrm{AB}$ \\
\hline$-\mathrm{N}$ & $13,7 \mathrm{AB}$ & $132,8 \mathrm{BC}$ & $99,0 \mathrm{CD}$ & $49,7 \mathrm{C}$ & $295,3 \mathrm{C}$ \\
\hline$-\mathrm{P}$ & $13,1 \mathrm{AB}$ & $292,9 \mathrm{~A}$ & $226,9 \mathrm{~A}$ & $115,1 \mathrm{AB}$ & $648,1 \mathrm{~A}$ \\
\hline$-\mathrm{K}$ & $11,3 \mathrm{AB}$ & $169,1 \mathrm{BC}$ & $113,7 \mathrm{CD}$ & $75,3 \mathrm{BC}$ & $369,4 \mathrm{BC}$ \\
\hline$-\mathrm{Ca}$ & $17,9 \mathrm{~A}$ & $170,7 \mathrm{BC}$ & $134,2 \mathrm{BC}$ & $82,3 \mathrm{BC}$ & $405,1 \mathrm{BC}$ \\
\hline$-\mathrm{Mg}$ & $7,2 \mathrm{~B}$ & $189,0 \mathrm{BC}$ & $66,1 \mathrm{D}$ & $41,7 \mathrm{C}$ & $304,1 \mathrm{C}$ \\
\hline$-\mathrm{S}$ & $14,9 \mathrm{AB}$ & $115,4 \mathrm{C}$ & $139,5 \mathrm{BC}$ & $85,0 \mathrm{BC}$ & $354,7 \mathrm{C}$ \\
\hline
\end{tabular}

Valores seguidos da mesma letra nas colunas não diferem significativamente pelo teste $\mathrm{T}$, a $5 \%$ de probabilidade. 
Quando se analisa o conteúdo de N, constata-se um baixo acúmulo nos ramos + caule e nas raízes das plantas do tratamento $-\mathrm{N}$, apesar de este valor continuar elevado nos filódios velhos e novos, que se equipara estatisticamente ao valor encontrado para o tratamento SC (Quadro 6). Apesar de não existir um decréscimo significativo nos conteúdos de $\mathrm{N}$ dos filódios de plantas submetidas ao tratamento $-\mathrm{N}$, observou-se queda na produção de matéria seca das plantas deste tratamento (Quadro 2). A falta de N na solução induziu a nodulação e a fixação eficiente de $\mathrm{N}_{2}$, no entanto o tempo decorrido até que passasse a ocorrer a fixação eficiente de $\mathrm{N}$ pode ter prejudicado a produção de biomassa das plantas do tratamento $-\mathrm{N}$.

As plantas que receberam tratamento com omissão de $\mathrm{K}$ apresentaram teores deste nutriente, nos filódios novos e velhos, cerca de dez vezes inferiores aos observados nas plantas que receberam solução nutritiva completa (Quadro 3). Também apresentaram os menores conteúdos deste nutriente em todas as suas partes (Quadro 7) comparadas a de todos os outros tratamentos. De modo geral, notou-se que a maior absorção de K ocorreu nos tratamentos $\mathrm{SC},-\mathrm{P} \mathrm{e}-\mathrm{Ca}$.

Os menores conteúdos de $\mathrm{Mg}$ foram observados nas plantas submetidas ao tratamento -Mg (Quadro 8). A ausência de $\mathrm{Ca}$ resultou em maior acúmulo de $\mathrm{Mg}$ nos filódios novos. Já nos filódios velhos, o maior conteúdo de $\mathrm{Mg}$ foi observado no tratamento -P, seguido do tratamento -Ca. Segundo Dias et al. (1994), ao analisar os teores de Mg nas diferentes partes de Acacia mangium, notou-se que não houve grande diferença em seus valores, evidenciando a elevada mobilidade interna do elemento. Este fato também foi observado em Acacia holosericea, para as plantas que receberam solução com omissão de $\mathrm{Mg}$, que apresentaram teor médio de $0,4 \mathrm{~g} / \mathrm{kg}$ de $\mathrm{Mg}$ tanto nos filódios novos quanto nos velhos (Quadro 3). O acúmulo total de Mg pelas plantas submetidas ao tratamento $-\mathrm{Mg}$ foi significativamente inferior ao valor apresentado pelas plantas de todos os outros tratamentos (Quadro 8).

O tratamento -S foi o que apresentou menor produção de matéria seca de filódios (Quadro 2). Não foi possível detectar, por meio da metodologia utilizada, o conteúdo de $\mathrm{S}$ no tratamento $-\mathrm{S}$ nos ramos + caule da planta (Quadro 9). Os conteúdos de $\mathrm{S}$ encontrados foram estatisticamente inferiores nas plantas do tratamento -S para todas as partes analisadas. Também foram constatados baixos conteúdos deste nutriente nos ramos + caule e nas raízes das plantas que receberam a solução $-\mathrm{Mg}$. O maior conteúdo de $\mathrm{S}$ nas plantas que receberam solução nutritiva completa foi observado nas raízes. Em estudos semelhantes, realizados com Acacia mangium, Dias et al. (1994) constataram que para todos os tratamentos existiu maior concentração de $\mathrm{S}$ nas raízes. As plantas submetidas à ausência de $\mathrm{S}$ acumularam cerca de 11 vezes menos $\mathrm{S}$ em seus tecidos do que aquelas que receberam a solução com todos os nutrientes (SC).

Quadro 6 - Conteúdo de N nos filódios novos (FN), nos filódios velhos (FV), nos ramos + caule ( $\mathrm{RC})$, nas raízes (RZ) e planta inteira de Acacia holosericea, em cada tratamento

Table $6-N$ content in new leaves ( FN), old leaves $(F V)$, branches + stem $(R C)$, roots $(R Z)$ and the whole plant of Acacia holosericea, in each treatment

\begin{tabular}{|l|c|c|c|c|c|}
\hline \multirow{2}{*}{$\begin{array}{l}\text { Trata- } \\
\text { mento }\end{array}$} & $\mathrm{FN}$ & $\mathrm{FV}$ & $\mathrm{RC}$ & $\mathrm{RZ}$ & Total \\
\cline { 2 - 6 } & \multicolumn{5}{|c|}{ (mg/planta) } \\
\hline $\begin{array}{c}\mathrm{SC} \\
-\mathrm{N}\end{array}$ & $64,1 \mathrm{AB}$ & $421,3 \mathrm{~A}$ & $118,1 \mathrm{BCD}$ & $231,0 \mathrm{~A}$ & $834,5 \mathrm{AB}$ \\
\hline$-\mathrm{A}$ & $36,4 \mathrm{AB}$ & $364,0 \mathrm{AB}$ & $83,7 \mathrm{DE}$ & $119,8 \mathrm{BC}$ & $633,9 \mathrm{~B}$ \\
\hline$-\mathrm{K}$ & $43,6 \mathrm{~B}$ & $375,1 \mathrm{AB}$ & $116,2 \mathrm{CD}$ & $123,7 \mathrm{BC}$ & $658,5 \mathrm{~B}$ \\
\hline$-\mathrm{Ca}$ & $86,7 \mathrm{~A}$ & $417,2 \mathrm{~A}$ & $144,2 \mathrm{ABC}$ & $172,9 \mathrm{AB}$ & $821,0 \mathrm{AB}$ \\
\hdashline$-\mathrm{Mg}$ & $34,5 \mathrm{~B}$ & $450,7 \mathrm{~A}$ & $48,4 \mathrm{E}$ & $68,9 \mathrm{C}$ & $602,4 \mathrm{~B}$ \\
\hline$-\mathrm{S}$ & $38,5 \mathrm{~B}$ & $230,1 \mathrm{~B}$ & $173,2 \mathrm{~A}$ & $156,7 \mathrm{ABC}$ & $598,5 \mathrm{~B}$ \\
\hline
\end{tabular}

Valores seguidos da mesma letra nas colunas não diferem significativamente pelo teste $\mathrm{T}$, a $5 \%$ de probabilidade.

Quadro 7 - Conteúdo de K nos filódios novos (FN), nos filódios velhos (FV), nos ramos + caule ( $\mathrm{RC}$ ), nas raízes (RZ) e planta inteira de Acacia holosericea, em cada tratamento

Table 7 - K content in new leaves $(F N)$, old leaves $(F V)$, branches + stem $(R C)$, roots $(R Z)$ and the whole plant of Acacia holosericea, in each treatment

\begin{tabular}{|l|c|c|c|c|c|}
\hline \multirow{2}{*}{$\begin{array}{l}\text { Trata- } \\
\text { mento }\end{array}$} & FN & FV & RC & RZ & Total \\
\cline { 2 - 6 } & \multicolumn{5}{|c|}{ (mg/planta) } \\
\hline $\mathrm{SC}$ & $45,0 \mathrm{~A}$ & $376,5 \mathrm{AB}$ & $210,2 \mathrm{~A}$ & $142,0 \mathrm{~A}$ & $773,6 \mathrm{~A}$ \\
\hline$-\mathrm{N}$ & $33,9 \mathrm{~A}$ & $279,6 \mathrm{BC}$ & $134,1 \mathrm{~B}$ & $70,8 \mathrm{BC}$ & $518,3 \mathrm{BC}$ \\
\hline$-\mathrm{P}$ & $29,3 \mathrm{AB}$ & $415,2 \mathrm{AB}$ & $227,6 \mathrm{~A}$ & $91,8 \mathrm{AB}$ & $763,9 \mathrm{~A}$ \\
\hline$-\mathrm{K}$ & $3,2 \mathrm{~B}$ & $27,5 \mathrm{D}$ & $3,8 \mathrm{D}$ & $4,6 \mathrm{D}$ & $39,1 \mathrm{D}$ \\
\hline$-\mathrm{Ca}$ & $57,3 \mathrm{~A}$ & $335,8 \mathrm{AB}$ & $192,8 \mathrm{~A}$ & $59,2 \mathrm{BC}$ & $645,1 \mathrm{AB}$ \\
\hline$-\mathrm{Mg}$ & $33,0 \mathrm{~A}$ & $436,9 \mathrm{~A}$ & $67,3 \mathrm{C}$ & $34,3 \mathrm{CD}$ & $371,5 \mathrm{ABC}$ \\
\hline$-\mathrm{S}$ & $30,2 \mathrm{AB}$ & $155,6 \mathrm{CD}$ & $125,8 \mathrm{~B}$ & $61,5 \mathrm{BC}$ & $373,1 \mathrm{C}$ \\
\hline
\end{tabular}

Valores seguidos da mesma letra nas colunas não diferem significativamente pelo teste $\mathrm{T}$, a $5 \%$ de probabilidade. 


\subsection{Sintomas Visuais de Deficiências Nutricionais Observados}

Os sintomas de deficiência nutricional começaram a se manifestar a partir do segundo mês após a aplicação dos tratamentos de omissão dos macronutrientes (Quadro 10, Figura 1).

O primeiro sintoma que pôde ser observado foi o de deficiência de N, o que evidencia a elevada demanda por

Quadro 8 - Conteúdo de $\mathrm{Mg}$ nos filódios novos (FN), nos filódios velhos (FV), nos ramos + caule (RC), nas raízes (RZ) e planta inteira de Acacia holosericea, em cada tratamento

Table 8 - Mg content in new leaves (FN), old leaves (FV), branches + stem $(R C)$, roots $(R Z)$ and the whole plant of Acacia holosericea, in each treatment

\begin{tabular}{|c|c|c|c|c|c|}
\hline \multirow{2}{*}{$\begin{array}{l}\text { Trata- } \\
\text { mento }\end{array}$} & FN & FV & $\mathrm{RC}$ & $\mathrm{RZ}$ & Total \\
\hline & \multicolumn{5}{|c|}{ (mg/planta) } \\
\hline $\mathrm{SC}$ & $3,6 \mathrm{~B}$ & $17,1 \mathrm{BC}$ & $12,0 \mathrm{~A}$ & $11,8 \mathrm{AB}$ & $44,5 \mathrm{AB}$ \\
\hline$-\mathrm{N}$ & $3,1 \mathrm{BC}$ & $15,6 \mathrm{BCD}$ & $6,6 \mathrm{~B}$ & 6,6 BCD & $32,0 \mathrm{BC}$ \\
\hline - & $2,4 \mathrm{BC}$ & $25,3 \mathrm{~A}$ & $12,0 \mathrm{~A}$ & 8,0 ABC & $47,7 \mathrm{~A}$ \\
\hline$-\mathrm{K}$ & $1,8 \mathrm{BC}$ & $11,4 \mathrm{CDE}$ & $9,3 \mathrm{AB}$ & $12,1 \mathrm{~A}$ & $34,5 \mathrm{ABC}$ \\
\hline$-\mathrm{Ca}$ & $6,8 \mathrm{~A}$ & $18,9 \mathrm{AB}$ & $11,2 \mathrm{~A}$ & $10,6 \mathrm{AB}$ & $47,4 \mathrm{~A}$ \\
\hline$-\mathrm{Mg}$ & $0,4 \mathrm{C}$ & $6,4 \mathrm{E}$ & $1,1 \mathrm{C}$ & $1,9 \mathrm{D}$ & $9,8 \mathrm{D}$ \\
\hline "'-S & $2,8 \mathrm{BC}$ & $9,7 \mathrm{DE}$ & $10,9 \mathrm{~A}$ & $5,0 \mathrm{CD}$ & $28,4 \mathrm{C}$ \\
\hline
\end{tabular}

Valores seguidos da mesma letra nas colunas não diferem significativamente pelo teste $\mathrm{T}$, a $5 \%$ de probabilidade. este nutriente de espécies da família Leguminosae. Inicialmente, houve clorose de filódios velhos, uma vez que o N é bastante móvel no floema e, como mencionado, compõe a molécula de clorofila, que é o pigmento verde mais importante no processo fotossintético (Devlin, 1969). No decorrer do tempo houve senescência e queda de todos os filódios velhos que apresentaram clorose, e o sintoma começou a manifestar-se com menor intensidade.

Quadro 9 - Conteúdo de $\mathrm{S}$ nos filódios novos (FN), nos filódios velhos (FV), nos ramos + caule ( $\mathrm{RC}$ ), nas raízes (RZ) e planta inteira de Acacia holosericea, em cada tratamento

Table 9 - S content in new leaves (FN), old leaves (FV), branches + stem $(R C)$, roots $(R Z)$ and the whole plant of Acacia holosericea, in each treatment

\begin{tabular}{|c|c|c|c|c|c|}
\hline \multirow{2}{*}{$\begin{array}{c}\text { Trata- } \\
\text { mento }\end{array}$} & FN & FV & RC & RZ & Total \\
\cline { 2 - 6 } & \multicolumn{5}{|c|}{$(\mathrm{mg} /$ planta) } \\
\hline $\mathrm{SC}$ & $2,6 \mathrm{AB}$ & $12,5 \mathrm{~B}$ & $8,0 \mathrm{~A}$ & $18,3 \mathrm{~A}$ & $41,5 \mathrm{~A}$ \\
\hline$-\mathrm{N}$ & $1,6 \mathrm{BC}$ & $10,5 \mathrm{~B}$ & $5,2 \mathrm{~B}$ & $7,8 \mathrm{BC}$ & $25,1 \mathrm{~B}$ \\
\hline$-\mathrm{P}$ & $1,3 \mathrm{BC}$ & $13,5 \mathrm{~B}$ & $8,5 \mathrm{~A}$ & $14,2 \mathrm{AB}$ & $37,6 \mathrm{AB}$ \\
\hline$-\mathrm{K}$ & $2,8 \mathrm{AB}$ & $10,4 \mathrm{~B}$ & $5,5 \mathrm{~B}$ & $11,9 \mathrm{AB}$ & $30,6 \mathrm{AB}$ \\
\hline$-\mathrm{Ca}$ & $3,7 \mathrm{~A}$ & $13,3 \mathrm{~B}$ & $8,8 \mathrm{~A}$ & $12,6 \mathrm{AB}$ & $38,4 \mathrm{~A}$ \\
\hline$-\mathrm{Mg}$ & $1,9 \mathrm{~B}$ & $21,8 \mathrm{~A}$ & $2,7 \mathrm{C}$ & $3,1 \mathrm{C}$ & $29,5 \mathrm{AB}$ \\
\hline$-\mathrm{S}$ & $0,1 \mathrm{C}$ & $1,2 \mathrm{C}$ & $\mathrm{ALR}^{\mathrm{I}}$ & $2,4 \mathrm{C}$ & $3,7 \mathrm{C}$ \\
\hline
\end{tabular}

Valores seguidos da mesma letra nas colunas não diferem significativamente pelo teste $\mathrm{T}$, a $5 \%$ de probabilidade.

1/ ALR: Abaixo do limite de resolução da metodologia.

Quadro 10 - Sintomas visuais de deficiência nutricional apresentados pelas plantas de Acacia holosericea sob a omissão de cada macronutriente

Table 10 - Visual symptoms of nutritional deficiency displayed by plants of Acacia holosericea under omission of each macronutrient

\begin{tabular}{|c|l|}
\hline Nutriente & \multicolumn{1}{|c|}{ Sintoma Visual da Deficiência } \\
\hline $\mathrm{N}$ & $\begin{array}{l}\text { Drástica redução na produção de matéria seca vegetal. Clorose de filódios mais velhos, começando pelas } \\
\text { extremidades até ocupar inteiramente o filódio, seguindo-se senescência e queda destes. }\end{array}$ \\
\hline $\mathrm{P} \mathrm{P}^{\mathrm{I}} \mathrm{K}$ & $\begin{array}{l}\text { Redução na produção de matéria seca vegetal. Necrose nas bordas dos filódios mais velhos, prolongando-se } \\
\text { pelas extremidades. }\end{array}$ \\
\hline $\mathrm{K}$ & $\begin{array}{l}\text { Pequena reduçãa na produçãão de matéria seca vegetal. Dobras e deformações dos filódios mais novos e e } \\
\text { gemas, com necrose das bordas. }\end{array}$ \\
\hline $\mathrm{Mg}$ & $\begin{array}{l}\text { Drástica redução na produção de matéria seca vegetal. Aparecimento de pontos arroxeados internervais } \\
\text { evoluindo para clorose internerval seguida de necrose. }\end{array}$ \\
\hline $\mathrm{S}$ & $\begin{array}{l}\text { Redução na produção de matéria seca vegetal. Clorose dos filódios mais novos e gemas e alteração na } \\
\text { arquitetura natural da planta, com os filódios apontados para o alto, ou seja, aproximando-se de uma posição } \\
\text { paralela ao caule (diminuição do ângulo de inserção dos filódios ao caule). }\end{array}$ \\
\hline
\end{tabular}

1/ As plantas que receberam o tratamento -P não apresentaram alterações visuais em seus filódios durante todo o período referente à aplicação dos tratamentos até a colheita do experimento. 


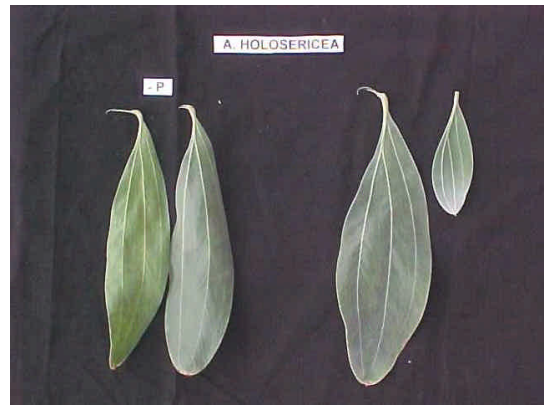

A

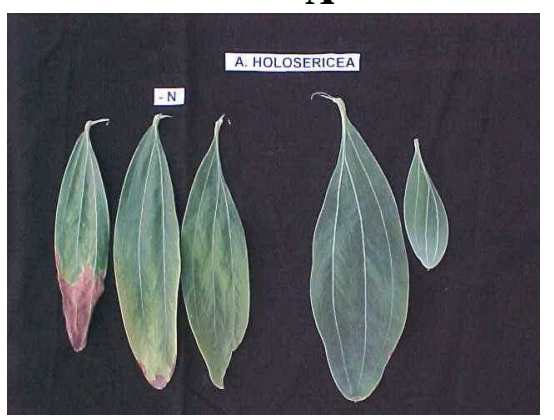

C

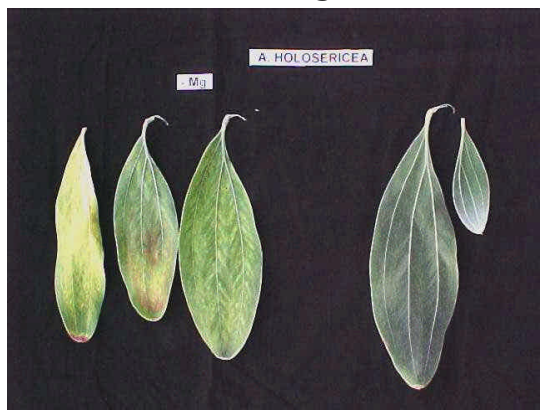

$\mathbf{E}$

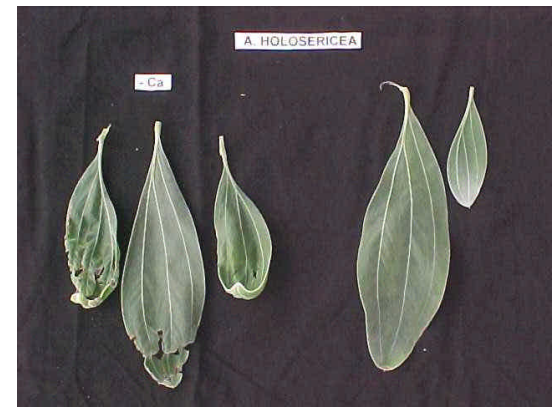

B

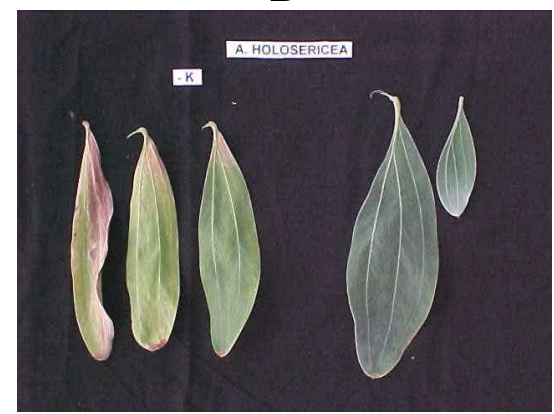

D

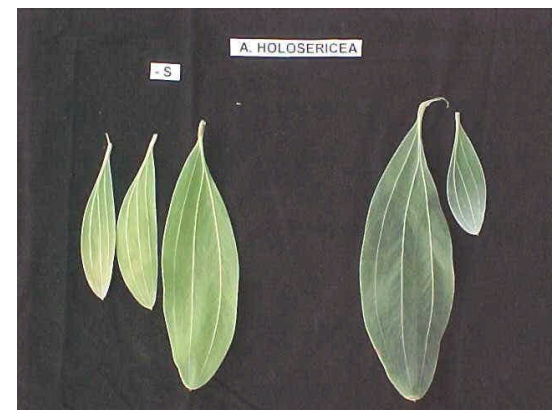

$\mathbf{F}$

Figura 1 - Sintomas de deficiência nutricional em filódios de A. holosericea em cada um dos tratamentos. Filódios à direita nas fotos são das plantas que receberam a solução nutritiva completa (SC). A) SC-P; B) SC-Ca; C) SC-N; D) SC-K; E) SC-Mg; F) SC-S.

Figure 1 - Nutritional deficiency symptoms in leaves of A. holosericea in each treatment. Leaves on the right side of the photographs refer to the plants that received the complete nutritive solution (SC). A) SC-P;B) $S C-C a ; C) S C-N$; D) $S C-K$; E) $S C-M g$; F) $S C-S$.

Ao final do experimento, os teores de $\mathrm{N}$ nos filódios das plantas do tratamento $-\mathrm{N}$ foram superiores aos das plantas que receberam solução nutritiva completa (Quadro 3). Este fato, acompanhado do gradual desaparecimento do sintoma de deficiência de N, foi principalmente devido à formação de nódulos nas raízes, promovendo a fixação simbiótica de $\mathrm{N}_{2}$.
O segundo sintoma de deficiência observado, que apareceu pouco depois do $\mathrm{N}$, foi o de deficiência de $\mathrm{Mg}$. Inicialmente, foram observadas manchas arroxeadas na região internerval dos filódios mais velhos, sintoma típico de deficiência de $\mathrm{P}$ para muitas espécies. No entanto, as manchas arroxeadas, que apareceram em todas as plantas que receberam o tratamento -Mg, evoluíram para a clorose 
internerval, o que, por sua vez, é um sintoma típico de deficiência de $\mathrm{Mg}$. A colheita do ensaio, seis meses após a aplicação dos tratamentos, foi tardia para as plantas do tratamento -Mg, que demonstraram grande sensibilidade à ausência deste nutriente, apresentando os menores valores de matéria seca de ramos + caule e de raízes, o que resultou no baixo porte destas plantas (Quadro 2).

Na ocasião da colheita, foi possível a detecção visual dos sintomas de deficiência de todos os macronutrientes, com exceção do P. Possivelmente, a reserva de P inorgânico foi suficiente para que não houvesse manifestação de deficiência nutricional de $\mathrm{P}$ nas plantas do tratamento -P durante todo o período de cultivo com supressão deste macronutriente.

A deficiência de $\mathrm{K}$ resultou em necrose nas bordas dos filódios mais velhos, prolongando-se pelas extremidades. Em Acacia mangium, o sintoma de deficiência de K manifestou-se de forma diferente, ocasionando clorose internerval e folhas quebradiças (Dias et al., 1994).

As plantas do tratamento -Ca apresentaram os filódios novos com deformações e dobras, além de necrose nas bordas. Essas alterações na estrutura dos filódios ocorrem devido à importância deste nutriente para a permeabilidade e o transporte na membrana e para a manutenção da integridade celular (Läuchli \& Bieleski, 1983).

O sintoma de deficiência de $\mathrm{S}$ mostrou-se, em parte, semelhante ao encontrado na literatura, com os filódios mais novos exibindo clorose geral. No entanto, em Acacia holosericea também ocorreu uma mudança na arquitetura da planta, com os filódios novos sendo lançados em um ângulo maior com relação à horizontal (apontados para cima) e também mais coriáceos.

Com exceção do N, os conteúdos dos demais macronutrientes nas plantas que receberam os tratamentos com a respectiva omissão foram inferiores àqueles encontrados para as plantas do tratamento SC, o que demonstra que os sintomas foliares observados foram, de fato, decorrentes de deficiência nutricional (Quadro 3).

\section{CONCLUSÕES}

O acúmulo de fósforo pelas plantas durante a fase inicial do ensaio não permitiu a observação de sintomas de deficiência para o nutriente, apesar do baixo conteúdo do nutriente nos tecidos das plantas submetidas à sua ausência. Portanto, conclui-se que a espécie estudada pode acumular e utilizar de forma bastante eficiente estoques de P inorgânico. Para os demais macronutrientes foram observados sintomas de deficiência nutricional.

Com exceção dos tratamentos - $\mathrm{P}$ e -Ca, a supressão dos outros macronutrientes provocou redução na produção de matéria seca total, especialmente a supressão de $\mathrm{N}$ e de $\mathrm{Mg}$.

A deficiência de $\mathrm{S}$ alterou a disposição natural dos filódios mais novos de Acacia holosericea.

\section{AGRADECIMENTOS}

Ao Conselho Nacional de Desenvolvimento Científico e Tecnológico - CNPq, pela concessão da bolsa de Iniciação Científica para a primeira autora.

À Mineração Rio do Norte S.A., por parte do suporte financeiro, necessário para a condução da pesquisa.

\section{REFERÊNCIAS BIBLIOGRÁFICAS}

ALVAREZ, V. et al. Métodos de análises de enxofre em solos e plantas. Viçosa: Editora UFV, 2001. 131 p.

BALIEIRO, F. C.; OLIVEIRA, I. G.; DIAS, L. E. Formação de mudas de Acacia holosericea e Acacia auriculiformis: resposta à calagem, fósforo, potássio e enxofre. Revista Árvore, v. 25, n. 2, p. 183-191, 2001.

BLANCHAR, R. W.; REHM, G.; CALDWELL, A. C. Sulfur in plant materials by digestion with nitric acid and perchloric acid. Soil Science Society America Proceedings, v. 29, p. 71-72, 1963.

BRAGA, J. M.; DEFELIPO, B. V. Determinação espectrofotométrica do fósforo em extrato de solos e plantas. Revista Ceres, v. 21, p. 73-85, 1974.

CAMPELLO, E. F. C. Utilização de leguminosas arbóreas fixadoras de nitrogênio na recuperação de áreas degradadas. 1998. $121 \mathrm{f}$. Tese (Doutorado em Ciência Florestal ) - Universidade Federal de Viçosa, Viçosa, 1998.

DEVLIN, R. M. Plant physiology. 2.ed. New York: Van Nostrand Reinhold Company, 1969. 262 p.

DIAS, L. E. et al. The use of nitrogen-fixing trees to revegetate bauxite and gold mined areas in the tropics: "Can trees substitute topsoil return?" In: BEIJING INTERNATIONAL SYMPOSIUM ON LAND RECLAMATION (ISLR), 1999, Beijing. Proceedings... Beijing: China Coal Industry Publishing House, 1999. p. 317-325. 
DIAS, L. E.; ALVAREZ V., V. H.; BRIENZA Jr., S. Formação de mudas de Acacia mangium. I. Resposta à calcário e fósforo. In: CONGRESSO FLORESTAL BRASILEIRO - SOCIEDADE BRASILEIRA DE SILVICULTURA E SOCIEDADE BRASILEIRA DE ENG. FLORESTAIS, 6., 1990, Campos do Jordão. Anais... Campos do Jordão: 1990. p. 449-453.

DIAS, L. E.; ALVAREZ V. V. H.; BRIENZA Jr., S. Formação de mudas de Acacia mangium. II. Resposta à nitrogênio e potássio. Rev. Árvore, Viçosa, v. 16, n. 2, p. 135-143. 1991b.

DIAS, L. E. et al. Formação de mudas de tachi-branco (Sclerolobium paniculatum Vogel). I. Resposta à calcário e fósforo. Pesquisa Agropecuária Brasileira, v. 26, n. 1, p. 69-76, 1991a.

DIAS, L. E. et al. Formação de mudas de taxi-branco (Sclerolobium paniculatum Vogel): Resposta a nitrogênio, potássio e enxofre. Revista Árvore, v. 16, n. 2, p. 135-143, 1992.

DIAS, L. E.; FARIA, S. M.; FRANCO, A. A. Crescimento de mudas de Acacia mangium Willd. em resposta à omissão de macronutrientes. Revista Árvore, v. 18, n. 2, p. 123-131, 1994.

FRANCO, A. A. et al. Nodulated legume trees for the recuperation of acid tropical soils. In: THE NORTH AMERICAN SYMBIOTIC NITROGEN FIXATION CONFERENCE, 12., 1989, Iowa. Proceedings... Iowa: Iowa State University, 1989. p. 70.
FRANCO, A. A.; DIAS, L. E.; FARIA, S. M. Uso de leguminosas florestais noduladas e micorrizadas como agentes de recuperação e manutenção da vida do solo: um modelo tecnológico. In: SIMPÓSIO SOBRE ESTRUTURA, FUNCIONAMENTO E MANEJO DE ECOSSISTEMAS, 1992, Rio de Janeiro. Resumos... Rio de Janeiro: UFRJ Inst. de Biologia, 1992. p. 93.

GRESPAN, S. L. Produção e eficiência nutricional de clones de eucalipto no norte do Espírito Santo e suas relações com características do solo. 1997. $81 \mathrm{f}$. Dissertação (Mestrado em Solos e Nutrição de Plantas) Universidade Federal de Viçosa, Viçosa, 1997.

JONES JR., J. B.; WOLF, B.; MILLS, H. A. Plant analysis handbook. A practical sampling, preparation, analysis, and interpretation guide. Athens: Micro-Macro Publishing Inc., 1991. $213 \mathrm{p}$.

LÄUCHLI, A.; BIELESKI, R. L. Inorganic plant nutrition. Berlin: Springer-Verlag, 1983. 449 p. (New Series, 15a).

RUIZ, H. A. Efeito do conteúdo de água sobre o transporte de fósforo em dois latossolos. 1986. $86 \mathrm{f}$. Tese (Doutorado em Solos e Nutrição de Plantas) Universidade Federal de Viçosa, Viçosa, 1986.

SIQUEIRA, J. O.; FRANCO, A. A. Biotecnologia do solo, fundamentos e perspectivas. Brasília: MEC/ABEAS; Lavras: ESA/FAEPE, 1988. 235 p.

THOMPSON, J. F.; SMITH, I. K.; MADISON, J. I. Sulphur metabolism in plants. In: TABATABAI, M. A. (Ed.). Sulfur in agriculture. Madison: America Society of Agronomy, 1986. p. 57-122. 